\title{
COMMUTATIVE COMBINATORIAL HOPF ALGEBRAS
}

\author{
FLORENT HIVERT, JEAN-CHRISTOPHE NOVELLI, AND JEAN-YVES THIBON
}

\begin{abstract}
We propose several constructions of commutative or cocommutative Hopf algebras based on various combinatorial structures, and investigate the relations between them. A commutative Hopf algebra of permutations is obtained by a general construction based on graphs, and its non-commutative dual is realized in three different ways, in particular as the Grossman-Larson algebra of heap ordered trees. Extensions to endofunctions, parking functions, set compositions, set partitions, planar binary trees and rooted forests are discussed. Finally, we introduce one-parameter families interpolating between different structures constructed on the same combinatorial objects.
\end{abstract}

\section{Contents}

1. Introduction 2

2. A commutative Hopf algebra of endofunctions 2

3. A commutative Hopf algebra of permutations 5

3.1. The Hopf algebra of bijective endofunctions 5

3.2. Duality 6

3.3. Cvclic tensors and $\mathfrak{S}$ OSum 7

3.4. Interpretation of $H(V)$

3.5. Subalgebras of $\mathfrak{S}$ OSum 10

3.6. Quotients of WSvm 12

3.7. The stalactic monoid 13

3.8. Other Hopf algebras derived from the stalactic congruence 14

4. Structure of SSym 18

4.1. A realization of SSym 18

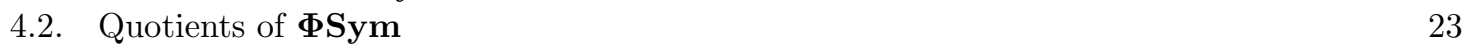

5. Parking functions and trees 23

5.1. A commutative algebra of parking functions 23

5.2. From labelled to unlabelled parking graphs 24

5.3. Binarv trees and nondecreasing parking functions 25

5.4. From nondecreasing parking functions to rooted forests 25

6. Quantum versions 25

6.1. Quantum quasi-svmmetric functions 25

6.2. Quantum free quasi-svmmetric functions 26

Acknowledgements 28

$\begin{array}{ll}\text { References } & 29\end{array}$ 


\section{INTRODUCTION}

Many examples of Hopf algebras based on combinatorial structures are known. Among these, algebras based on permutations and planar binary trees play a prominent role, and arise in seemingly unrelated contexts [21, 19, 8, 5]. As Hopf algebras, both are noncommutative and non cocommutative, and in fact self-dual.

More recently, cocommutative Hopf algebras of binary trees and permutations have been constructed 22, 22. In 22, binary trees arise as sums over rearrangements classes in an algebra of parking functions, while in [2], cocommutative Hopf algebras are obtained as the graded coalgebras associated with coradical fitrations.

In [25], a general method for constructing commutative Hopf algebras based on various kind of graphs has been presented. The aim of this note is to investigate Hopf algebras based on permutations and trees constructed by the method developed in [25. These commutative algebras are, by definition, realized in terms of polynomials in an infinite set of doubly indexed indeterminates. The dual Hopf algebras are then realized by means of non commutative polynomials in variables $a_{i j}$. We show that these first resulting algebras are isomorphic (in a non trivial way) to the duals of those of [2], and study some generalizations such as endofunctions, parking functions, set partitions, trees, forests, and so on.

The possibility to obtain in an almost systematic way commutative, and in general non cocommutative, versions of the usual combinatorial Hopf algebras leads us to conjecture that these standard versions should be considered as some kind of quantum groups, i.e., can be incorporated into one-parameter families, containing an enveloping algebra and its dual for special values of the parameter. A few results supporting this point of view are presented in the final section.

In all the paper, $\mathbb{K}$ will denote a field of characteristic zero. All the notations used here is as in [9, 25]. This paper is an expanded and updated version of the preprint [14.

\section{A commutative Hopf algebra of endofunctions}

Permutations can be regarded in an obvious way as labelled and oriented graphs whose connected components are cycles. Actually, arbitrary endofunctions (functions from $[n]:=\{1, \ldots, n\}$ to itself) can be regarded as labelled graphs, connecting $i$ with $f(i)$ for all $i$ so as to fit in the framework of [25], where a general process for building Hopf algebras of graphs is described.

In the sequel, we identify an endofunction $f$ of $[n]$ with the word

$$
w_{f}=f(1) f(2) \cdots f(n) \in[n]^{n} .
$$

Let $\left\{x_{i j} \mid i, j \geq 1\right\}$ be an infinite set of commuting indeterminates, and let $\mathcal{J}$ be the ideal of $R=\mathbb{K}\left[x_{i j} \mid i, j \geq 1\right]$ generated by the relations

$$
x_{i j} x_{i k}=0 \text { for all } i, j, k \text {. }
$$

For an endofunction $f:[n] \rightarrow[n]$, define

$$
M_{f}:=\sum_{i_{1}<\cdots<i_{n}} x_{i_{1} i_{f(1)}} \cdots x_{i_{n} i_{f(n)}},
$$


in $R / \mathcal{J}$.

It follows from [25], Section 4, that

Theorem 2.1. The $M_{f}$ span a subalgebra EQSym of the commutative algebra $R / \mathcal{J}$. More precisely, there exist non-negative integers $C_{f, g}^{h}$ such that

$$
M_{f} M_{g}=\sum_{h} C_{f, g}^{h} M_{h}
$$

\section{Example 2.2.}

$$
\begin{gathered}
M_{1} M_{22}=M_{133}+M_{323}+M_{223} \\
M_{1} M_{331}=M_{1442}+M_{4241}+M_{4431}+M_{3314} \\
M_{12} M_{21}=M_{1243}+M_{1432}+M_{4231}+M_{1324}+M_{3214}+M_{2134} \\
M_{12} M_{22}=M_{1244}+M_{1434}+M_{4234}+M_{1334}+M_{3234}+M_{2234} \\
M_{12} M_{133}=3 M_{12355}+2 M_{12445}+2 M_{12545}+M_{13345}+M_{14345}+M_{15345}
\end{gathered}
$$

The shifted concatenation of two endofunctions $f:[n] \rightarrow[n]$ and $g:[m] \rightarrow[m]$ is the endofunction $h:=f \bullet g$ of $[n+m]$ such that $w_{h}:=w_{f} \bullet w_{g}$, that is

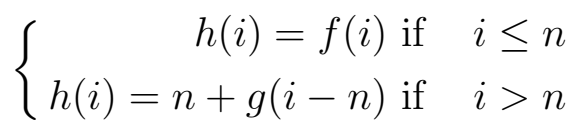

We can now give a combinatorial interpretation of the coefficient $C_{f, g}^{h}$ : if $f:[n] \rightarrow$ $[n]$ and $g:[m] \rightarrow[m]$, this coefficient is the number of permutations $\tau$ in the shuffle product $(1 \ldots p) \amalg(p+1 \ldots p+n)$ such that

$$
h=\tau^{-1} \circ(f \bullet g) \circ \tau .
$$

For example, with $f=12$ and $g=22$, one finds the set (see Equation (8))

$$
\{1244,1434,4234,1334,3234,2234\} \text {. }
$$

Now, still following [25], define a coproduct by

$$
\Delta M_{h}:=\sum_{(f, g) ; f \bullet g=h} M_{f} \otimes M_{g} .
$$

This endows EQSym with a (commutative, non cocommutative) Hopf algebra structure. 


\section{Example 2.3.}

$$
\begin{gathered}
\Delta M_{626124}=M_{626124} \otimes 1+1 \otimes M_{626124} . \\
\Delta M_{4232277}=M_{4232277} \otimes 1+M_{42322} \otimes M_{22}+1 \otimes M_{4232277 .}
\end{gathered}
$$

Define a connected endofunction as a function that cannot be obtained by non trivial shifted concatenation. For example, the connected endofunctions for $n=$ $1,2,3$ are

$$
\text { 1, } \quad 11,21,22 \text {, }
$$$$
111,112,121,131,211,212,221,222,231,232 \text {, }
$$$$
233,311,312,313,321,322,323,331,332,333 \text {, }
$$

and the generating series of their number begins with

$$
t+3 t^{2}+20 t^{3}+197 t^{4}+2511 t^{5}+38924 t^{6}+708105 t^{7}+14769175 t^{8} .
$$

Then, the definition of the coproduct of the $M_{f}$ implies

Proposition 2.4. If $\left(\mathbf{S}^{f}\right)$ denotes the dual basis of $\left(M_{f}\right)$, the graded dual $\mathbf{E S y m}:=$ EQSym* is free over the set

$$
\left\{\mathbf{S}^{f} \mid f \text { connected }\right\} .
$$

Indeed, Equation (13) is equivalent to

$$
\mathbf{S}^{f} \mathbf{S}^{g}=\mathbf{S}^{f \bullet g} .
$$

Now, ESym being a graded connected cocommutative Hopf algebra, it follows from the Cartier-Milnor-Moore theorem that

$$
\mathbf{E S y m}=U(L),
$$

where $L$ is the Lie algebra of its primitive elements. Let us now prove

Theorem 2.5. As a graded Lie algebra, the primitive Lie algebra $L$ of $\mathbf{E S y m}$ is free over a set indexed by connected endofunctions.

Proof - Assume it is the case. By standard arguments on generating series, one finds that the number of generators of $L$ in degree $n$ is equal to the number of algebraic generators of ESym in degree $n$, parametrized for example by connected endofunctions. We will now show that $L$ has at least this number of generators and that those generators are algebraically independent, determining completely the dimensions of the homogeneous components $L_{n}$ of $L$ whose generating series begins by

$$
t+3 t^{2}+23 t^{3}+223 t^{4}+2800 t^{5}+42576 t^{6}+763220 t^{7}+15734388 t^{8}+\ldots
$$


Following Reutenauer [28] p. 58, denote by $\pi_{1}$ the Eulerian idempotent, that is, the endomorphism of ESym defined by $\pi_{1}=\log ^{*}(I d)$. It is obvious, thanks to the definition of $\mathbf{S}^{f}$ that

$$
\pi_{1}\left(\mathbf{S}^{f}\right)=\mathbf{S}^{f}+\cdots,
$$

where the dots stand for terms $\mathbf{S}^{g}$ such that $g$ is not connected. Since the $\mathbf{S}^{f}$ associated with connected endofunctions are independent, the dimension of $L_{n}$ is at least equal to the number of connected endofunctions of size $n$. So $L$ is free over a set of primitive elements parametrized by connected endofunctions.

There are many Hopf subalgebras of EQSym which can be defined by imposing natural restrictions to maps: being bijective (see Section 3), idempotent $\left(f^{2}=f\right.$ ), involutive $\left(f^{2}=i d\right)$, or more generally the Burnside classes $\left(f^{p}=f^{q}\right)$, and so on. We shall start with the Hopf algebra of permutations.

\section{A commutative Hopf algebra of Permutations}

We will use two different notations for permutations depending whether they are considered as bijections from $[1, n]$ onto itself or as product of cycles. In the first case, we will write $\sigma=31542$ for the bijection $\sigma$ where $\sigma(i)=\sigma_{i}$. In the second case, the same permutation will be written $\sigma=(1352)(4)$ since 31542 is composed of two cycles : the cycle (1352) sending each element to the next one (circularly) in the sequence and the cycle (4) composed of only one element.

3.1. The Hopf algebra of bijective endofunctions. Let us define $\mathfrak{S}$ QSYm as the subalgebra of EQSym spanned by the

$$
M_{\sigma}=\sum_{i_{1}<\cdots<i_{n}} x_{i_{1} i_{\sigma(1)}} \cdots x_{i_{n} i_{\sigma(n)}}
$$

where $\sigma$ runs over bijective endofunctions, i.e., permutations. Note that $\mathfrak{S} Q S y m$ is also isomorphic to the image of EQSym in the quotient of $R / \mathcal{J}$ by the relations

$$
x_{i k} x_{j k}=0 \text { for all } i, j, k \text {. }
$$

By the usual argument, it follows that

Proposition 3.1. The $M_{\sigma}$ span a Hopf subalgebra $\mathfrak{S} Q S y m$ of the commutative Hopf algebra EQSym.

As already mentionned, there exist non-negative integers $C_{\alpha, \beta}^{\gamma}$ such that

$$
M_{\alpha} M_{\beta}=\sum_{\gamma} C_{\alpha, \beta}^{\gamma} M_{\gamma}
$$

The combinatorial interpretation of the coefficients $C_{f, g}^{h}$ seen in Section 2 can be reformulated in the special case of permutations. Write $\alpha$ and $\beta$ as a union of disjoint cycles. Split the set $[n+m]$ into a set $A$ of $n$ elements, and its complement $B$, in all possible ways. For each splitting, apply to $\alpha$ (resp. $\beta$ ) in $A$ (resp. $B$ ) the unique 
increasing morphism of alphabets from $[n]$ to $A$ (resp. from $[m]$ to $B$ ) and return the list of permutations with the resulting cycles. On the example $\alpha=(1)(2)=12$ and $\beta=(13)(2)=321$, this yields

$$
\begin{aligned}
& (1)(2)(53)(4),(1)(3)(52)(4),(1)(4)(52)(3),(1)(5)(42)(3),(2)(3)(51)(4), \\
& (2)(4)(51)(3),(2)(5)(41)(3),(3)(4)(51)(2),(3)(5)(41)(2),(4)(5)(31)(2) .
\end{aligned}
$$

This set corresponds to the permutations and multiplicities of Equation (31).

A third interpretation of this product comes from the dual coproduct point of view:

$C_{\alpha, \beta}^{\gamma}$ is the number of ways of getting $(\alpha, \beta)$ as the standardized words of pairs $(a, b)$ of two complementary subsets of cycles of $\gamma$. For example, with $\alpha=12, \beta=321$, and $\gamma=52341$, one has three solutions for the pair $(a, b)$, namely

$$
((2)(3),(4)(51)), \quad((2)(4),(3)(51)), \quad((3)(4),(2)(51)),
$$

which is coherent with Equations (26) and (31).

\section{Example 3.2.}

$$
\begin{gathered}
M_{12 \cdots n} M_{12 \cdots p}=\left(\begin{array}{c}
n+p \\
n
\end{array}\right) M_{12 \cdots(n+p)} \\
M_{1} M_{21}=M_{132}+M_{213}+M_{321} \\
M_{12} M_{21}=M_{1243}+M_{1324}+M_{1432}+M_{2134}+M_{3214}+M_{4231} \\
M_{12} M_{321}=M_{12543}+M_{14325}+2 M_{15342}+M_{32145}+2 M_{42315}+3 M_{52341} \\
M_{21} M_{123}=M_{12354}+M_{12435}+M_{12543}+M_{13245}+M_{14325} \\
+M_{15342}+M_{21345}+M_{32145}+M_{42315}+M_{52341} \\
M_{21} M_{231}=M_{21453}+M_{23154}+M_{24513}+M_{25431}+M_{34152} \\
+M_{34521}+M_{35412}+M_{43251}+M_{43512}+M_{53421} .
\end{gathered}
$$

3.2. Duality. Recall that the coproduct is given by

$$
\Delta M_{\sigma}:=\sum_{(\alpha, \beta) ; \alpha \bullet \beta=\sigma} M_{\alpha} \otimes M_{\beta} .
$$

As in Section 2, this implies

Proposition 3.3. If $\left(\mathbf{S}^{\sigma}\right)$ denotes the dual basis of $\left(M_{\sigma}\right)$, the graded dual $\mathfrak{S S y m}:=$ $\mathfrak{S}$ QSym* ${ }^{*}$ is free over the set

$$
\left\{\mathbf{S}^{\alpha} \mid \alpha \text { connected }\right\}
$$


Indeed, Equation (34) is equivalent to

$$
\mathbf{S}^{\alpha} \mathbf{S}^{\beta}=\mathbf{S}^{\alpha \bullet \beta} \text {. }
$$

Note that $\mathfrak{S S y m}$ is both a subalgebra and a quotient of ESym, since $\mathfrak{S}$ QSYm is both a quotient and a subalgebra of EQSym.

Now, as before, SSym being a graded connected cocommutative Hopf algebra, it follows from the Cartier-Milnor-Moore theorem that

$$
\mathfrak{S S y m}=U(L)
$$

where $L$ is the Lie algebra of its primitive elements.

The same argument as in Section 2 proves

Theorem 3.4. The graded Lie algebra $L$ of primitive elements of $\mathbf{S S y m}$ is free over a set indexed by connected permutations.

Corollary 3.5. SSym is isomorphic to $H_{O}$, the Grossman-Larson Hopf algebra of heap-ordered trees [1].

According to [2], SQSym $\left(=\mathfrak{S S y m}^{*}\right)$ is therefore isomorphic to the quotient of FQSym by its coradical filtration.

3.3. Cyclic tensors and $\mathfrak{S} Q S y m$. For a vector space $V$, let $\Gamma^{n} V$ be the subspace of $V^{\otimes n}$ spanned by cyclic tensors, i.e., sums of the form

$$
\sum_{k=0}^{n-1}\left(v_{1} \otimes \cdots \otimes v_{n}\right) \gamma^{k}
$$

where $\gamma$ is the cycle $(1,2, \ldots, n)$, the right action of permutations on tensors being as usual

$$
\left(v_{1} \otimes \cdots \otimes v_{n}\right) \sigma=v_{\sigma(1)} \otimes \cdots \otimes v_{\sigma(n)} .
$$

Clearly, $\Gamma^{n} V$ is stable under the action of $G L(V)$, and its character is the symmetric function "cyclic character" [30, 17]:

$$
l_{n}^{(0)}=\frac{1}{n} \sum_{d \mid n} \phi(d) p_{d}^{n / d},
$$

where $\phi$ is Euler's function.

Let $L_{n}^{(0)}$ be the subspace of $\mathbb{C S}_{n}$ spanned by cyclic permutations. This is a submodule of $\mathbb{C S}_{n}$ for the conjugation action $\rho_{\tau}(\sigma)=\tau \sigma \tau^{-1}$ with Frobenius characteristic $l_{n}^{(0)}$. Then one can define the analytic functor $\Gamma[15,20]$ :

$$
\Gamma(V)=\sum_{n \geq 0} V^{\otimes n} \otimes_{\mathbb{C S}_{n}} L_{n}^{(0)} .
$$

Let $\bar{\Gamma}(V)=\bigoplus_{n \geq 1} \Gamma^{n}(V)$. Its symmetric algebra $H(V)=S(\bar{\Gamma}(V))$ can be endowed with a Hopf algebra structure, by declaring the elements of $\bar{\Gamma}(V)$ primitive. 
As an analytic functor, $V \mapsto H(V)$ corresponds to the sequence of $\mathfrak{S}_{n}$-modules $M_{n}=\mathbb{C S}_{n}$ endowed with the conjugation action, that is,

$$
H(V)=\bigoplus_{n \geq 0} V^{\otimes n} \otimes_{\mathbb{C S}_{n}} M_{n}
$$

so that basis elements of $H_{n}(V)$ can be identified with symbols $\left[\begin{array}{l}w \\ \sigma\end{array}\right]$ with $w \in V^{\otimes n}$ and $\sigma \in \mathfrak{S}_{n}$ subject to the equivalences

$$
\left[\begin{array}{c}
w \tau^{-1} \\
\tau \sigma \tau^{-1}
\end{array}\right] \equiv\left[\begin{array}{c}
w \\
\sigma
\end{array}\right]
$$

Let $A:=\left\{a_{n} \mid n \geq 1\right\}$ be an infinite linearly ordered alphabet, and $V=\mathbb{C} A$. We identify a tensor product of letters $a_{i_{1}} \otimes \cdots \otimes a_{i_{n}}$ with the corresponding word $w=$ $a_{i_{1}} \ldots a_{i_{n}}$ and denote by $(w) \in \Gamma^{n} V$ the circular class of $w$. A basis of $H_{n}(V)$ is then given by the commutative products

$$
\underline{m}=\left(w_{1}\right) \cdots\left(w_{p}\right) \in S(\bar{\Gamma} V)
$$

of circular words, with $\left|w_{1}\right|+\cdots+\left|w_{p}\right|=n$ and $\left|w_{i}\right| \geq 1$ for all $i$.

With such a basis element, we can associate a permutation by the following standardization process. Fix a total order on circular words, for example the lexicographic order on minimal representatives. Write $\underline{m}$ as a non-decreasing product

$$
\underline{m}=\left(w_{1}\right) \cdots\left(w_{p}\right) \text { with }\left(w_{1}\right) \leq\left(w_{2}\right) \leq \cdots \leq\left(w_{p}\right),
$$

and compute the ordinary standardization $\sigma^{\prime}$ of the word $w=w_{1} \cdots w_{p}$. Then, $\sigma$ is the permutation obtained by parenthesing the word $\sigma^{\prime}$ like $\underline{m}$ and interpreting the factors as cycles. For example, if

$$
\begin{aligned}
\underline{m} & =(c b a)(a b a)(a c)(b a)=(a a b)(a b)(a c)(a c b) \\
w & =a a b a b a c a c b \\
\sigma^{\prime} & =12637495108 \\
\sigma & =(126)(37)(49)(5108) \\
\sigma & =(2,6,7,9,10,1,3,5,4,8)
\end{aligned}
$$

We set $\sigma=\operatorname{cstd}(\underline{m})$ and define it as the circular standardized of $\underline{m}$.

Let $H_{\sigma}(V)$ be the subspace of $H_{n}(V)$ spanned by those $\underline{m}$ such that $\operatorname{cstd}(\underline{m})=$ $\sigma$, and let $\pi_{\sigma}: H(V) \rightarrow H_{\sigma}(V)$ be the projector associated with the direct sum decomposition

$$
H(V)=\bigoplus_{n \geq 0} \bigoplus_{\sigma \in \mathfrak{S}_{n}} H_{\sigma}(V)
$$

Computing the convolution of such projectors then yields the following

Theorem 3.6. The $\pi_{\sigma}$ span a subalgebra of the convolution algebra End $^{\mathrm{gr}} H(V)$, isomorphic to $\mathfrak{S}$ QSym via $\pi_{\sigma} \mapsto M_{\sigma}$. 
Proof - First, note that $\pi_{\alpha} * \pi_{\beta}$ is a sum of $\pi_{\gamma}$ : indeed,

$$
\pi_{\alpha} * \pi_{\beta}(\underline{m})=\pi_{\alpha} * \pi_{\beta}(\operatorname{cstd}(\underline{m})),
$$

since the circular standardized of a subword of $\underline{m}$ is equal to the circular standardized of the same subword of $\operatorname{cstd}(\underline{m})$. So

$$
\pi_{\alpha} * \pi_{\beta}=\sum_{\sigma} D_{\alpha, \beta}^{\sigma} \pi_{\sigma}
$$

Now, by definition of $\pi_{\alpha}$ and by the third interpretation of the product of the $M_{\sigma}$, one concludes that $D_{\alpha, \beta}^{\sigma}$ is equal to the $C_{\alpha, \beta}^{\sigma}$ of Equation (25).

3.4. Interpretation of $H(V)$. The Hopf algebra $H(V)$ can be interpreted as an algebra of functions as follows. Assume $V$ has dimension $d$, and let $a_{1}, \ldots, a_{d}$ be a basis of $V$.

To the generator $\left(a_{i_{1}} \cdots a_{i_{n}}\right)$ of $H(V)$, we associate the function of $d$ matrices

$$
f_{\left(i_{1}, \ldots, i_{n}\right)}\left(A_{1}, \ldots, A_{d}\right)=\operatorname{tr}\left(A_{i_{1}} \cdots A_{i_{n}}\right) .
$$

These functions are clearly invariant under simultaneous conjugation $A_{i} \mapsto M A_{i} M^{-1}$, and it is easy to prove that they generate the ring of invariants of $G L(N, \mathbb{C})$ in the symmetric algebra

$$
M_{N}(\mathbb{C})^{\oplus d} \simeq M_{N}(\mathbb{C}) \otimes V
$$

Indeed, let us set $U=\mathbb{C}^{N}$ and identify $M_{N}(\mathbb{C})$ with $U \otimes U^{*}$. Then, the character of $G L(U)$ in $S^{n}\left(U \otimes U^{*} \otimes V\right)$ is $h_{n}\left(X X^{\vee} N\right)$, where $X=\sum x_{i}, X^{\vee}=\sum x_{i}^{-1}$.

By the Cauchy formula,

$$
h_{n}\left(X X^{\vee} N\right)=\sum_{\lambda \vdash n} s_{\lambda}(N X) s_{\lambda}\left(X^{\vee}\right),
$$

and the dimension of the invariant subspace is

$$
\begin{aligned}
\operatorname{dim} S^{n}\left(U \otimes U^{*} \otimes V\right)^{G L(U)} & =\left\langle h_{n}\left(X X^{\vee} N\right), 1\right\rangle_{G L(U)} \\
& =\sum_{\lambda \vdash n}\left\langle s_{\lambda}(N X), s_{\lambda}(X)\right\rangle \\
& =\sum_{\lambda, \mu \vdash n} s_{\lambda} * s_{\mu}(N)\left\langle s_{\lambda}, s_{\mu}\right\rangle \\
& =\sum_{\lambda \vdash n}\left(s_{\lambda} * s_{\lambda}\right)(N) .
\end{aligned}
$$

The characteristic of the conjugation action of $\mathfrak{S}_{n}$ on $\mathbb{C S}_{n}$ is precisely $\sum_{\lambda \vdash n}\left(s_{\lambda} * s_{\lambda}\right)$, so this is the dimension of $H_{n}(V)$. We have therefore established:

Theorem 3.7. Let $F_{N}^{(d)}$ be the algebra of $G L(N, \mathbb{C})$-invariant polynomial functions on $M_{N}(\mathbb{C})^{\oplus d} \simeq M_{N}(\mathbb{C}) \otimes V$, endowed with the comultiplication

$$
\Delta f\left(A_{1}^{\prime}, \ldots, A_{d}^{\prime} ; A_{1}^{\prime \prime}, \ldots, A_{d}^{\prime \prime}\right):=f\left(A_{1}^{\prime} \oplus A_{1}^{\prime \prime}, \ldots, A_{d}^{\prime} \oplus A_{d}^{\prime \prime}\right)
$$


Then the map $\left(a_{i_{1}} \cdots a_{i_{k}}\right) \mapsto f_{\left(i_{1}, \ldots, i_{k}\right)}$ is an epimorphism of bialgebras $H(V) \rightarrow F_{N}^{(d)}$.

\subsection{Subalgebras of $\mathfrak{S} Q S y m$.}

3.5.1. Symmetric functions in noncommuting variables (dual). For a permutation $\sigma \in \mathfrak{S}_{n}$, let $\operatorname{supp}(\sigma)$ be the partition $\pi$ of the set $[n]$ whose blocks are the supports of the cycles of $\sigma$. The sums

$$
U_{\pi}:=\sum_{\operatorname{supp}(\sigma)=\pi} M_{\sigma}
$$

span a Hopf subalgebra $\Pi Q S y m$ of $\mathfrak{S} Q S y m$, which is isomorphic to the graded dual of the Hopf algebra of symmetric functions in noncommuting variables (such as in [29, 4, not to be confused with $\mathbf{S y m})$, which we will denote here by $\mathbf{W} \operatorname{Sym}(A)$, for Word symmetric functions. Indeed, from the product rule of the $M_{\sigma}$ given in Equation (25), one easily finds

$$
U_{\pi^{\prime}} U_{\pi^{\prime \prime}}:=\sum C_{\pi^{\prime}, \pi^{\prime \prime}}^{\pi} U_{\pi}
$$

where $C_{\pi^{\prime}, \pi^{\prime \prime}}^{\pi}$ is the number of ways of splitting the parts of $\pi$ into two subpartitions whose standardized words are $\pi^{\prime}$ and $\pi^{\prime \prime}$. For example,

$$
\begin{aligned}
U_{\{\{1,2,4\},\{3\}\}} U_{\{\{1\}\}} & =U_{\{\{1,2,4\},\{3\},\{5\}\}}+2 U_{\{\{1,2,5\},\{3\},\{4\}\}} \\
& +U_{\{\{1,3,5\},\{4\},\{2\}\}}+U_{\{\{2,3,5\},\{4\},\{1\}\}} .
\end{aligned}
$$

The dual WSym $(A)$ of $\Pi Q S y m$ is the subspace of $\mathbb{K}\langle A\rangle$ spanned by the orbits of $\mathfrak{S}(A)$ on $A^{*}$. These orbits are naturally labelled by set partitions of $[n]$, the orbit corresponding to a partition $\pi$ being constituted of the words

$$
w=a_{1} \ldots a_{n},
$$

such that $a_{i}=a_{j}$ iff $i$ and $j$ are in the same block of $\pi$. The sum of these words will be denoted by $\mathbf{M}_{\pi}$.

For example,

$$
\mathbf{M}_{\{\{1,3,6\},\{2\},\{4,5\}\}}:=\sum_{a \neq b ; b \neq c ; a \neq c} a b a c c a .
$$

It is known that the natural coproduct of WSym (given as usual by the ordered sum of alphabets) is cocommutative [4 and that WSym is free over connected set partitions. The same argument as in Theorem 2.5 shows that $\Pi Q S y m^{*}$ is free over the same graded set, hence that MQSym is indeed isomorphic to WSym*.

3.5.2. Quasi-symmetric functions. One can also embed QSym into $\Pi Q S y m$ : take as total ordering on finite sets of integers $\left\{i_{1}<\cdots<i_{r}\right\}$ the lexicographic order on the words $\left(i_{1}, \ldots, i_{r}\right)$. Then, any set partition $\pi$ of $[n]$ has a canonical representative $B$ as a non-decreasing sequence of blocks $\left(B_{1} \leq B_{2} \leq \cdots \leq B_{r}\right)$. Let $I=K(\pi)$ be the composition $\left(\left|B_{1}\right|, \ldots,\left|B_{r}\right|\right)$ of $n$. The sums

$$
U_{I}:=\sum_{K(\pi)=I} U_{\pi}=\sum_{K(\sigma)=I} M_{\sigma}
$$


where $K(\sigma)$ denotes the ordered cycle type of $\sigma$, span a Hopf subalgebra of $\Pi Q S y m$ and $\mathfrak{S} Q S y m$, which is isomorphic to QSym. Indeed, from the product rule of the $M_{\sigma}$ given in Equation (25), one easily finds

$$
U_{I^{\prime}} U_{I^{\prime \prime}}:=\sum_{I} C_{I^{\prime}, I^{\prime \prime}}^{I} U_{I}
$$

where $C_{I^{\prime}, I^{\prime \prime}}^{I}$ is the coefficient of $I$ in $I^{\prime} \amalg I^{\prime \prime}$. For example,

$$
\begin{aligned}
U_{(1,3,1)} U_{(1,2)}= & 2 U_{(1,1,2,3,1)}+2 U_{(1,1,3,1,2)}+2 U_{(1,1,3,2,1)} \\
& +U_{(1,2,1,3,1)}+2 U_{(1,3,1,1,2)}+U_{(1,3,1,2,1)} .
\end{aligned}
$$

3.5.3. Symmetric functions. Furthemore, if we denote by $\Lambda(I)$ the partition associated with a composition $I$ by sorting $I$ and by $\Lambda(\pi)$ the partition $\lambda$ whose parts are the sizes of the blocks of $\pi$, the sums

$$
u_{\lambda}:=\sum_{\Lambda(I)=\lambda} U_{I}=\sum_{\Lambda(\pi)=\lambda} U_{\pi}=\sum_{Z(\sigma)=\lambda} M_{\sigma}
$$

where $Z(\sigma)$ denotes the cycle type of $\sigma$, span a Hopf subalgebra of QSym, ПQSym, and $\mathfrak{S} Q S y m$, which is isomorphic to Sym (ordinary symmetric functions). As an example of the product, one has

$$
u_{(3,3,2,1)} u_{(3,1,1)}=9 u_{(3,3,3,2,1,1,1)} .
$$

Indeed, it follows from Equation (61) that an explicit Hopf embedding of Sym into $\mathfrak{S}$ QSym is given by

$$
j: p_{\lambda}^{*} \rightarrow u_{\lambda}
$$

where $p_{\lambda}^{*}=\frac{p_{\lambda}}{z_{\lambda}}$ is the adjoint basis of products of power sums. The images of the usual generators of Sym under this embedding have simple expressions in terms of the infinite matrix $X=\left(x_{i j}\right)_{i, j \leq 1}$ :

$$
j\left(p_{n}\right)=\operatorname{tr}\left(X^{n}\right)
$$

which implies that $j\left(e_{n}\right)$ is the sum of the diagonal minors of order $n$ of $X$ :

$$
j\left(e_{n}\right)=\sum_{i_{1}<\cdots<i_{n}} \sum_{\sigma \in \mathfrak{S}_{n}} \varepsilon(\sigma) x_{i_{1} i_{\sigma(1)}} \ldots x_{i_{n} i_{\sigma(n)}}
$$

and $j\left(h_{n}\right)$ is the sum of the same minors of the permanent

$$
j\left(h_{n}\right)=\sum_{i_{1}<\cdots<i_{n}} \sum_{\sigma \in \mathfrak{S}_{n}} x_{i_{1} i_{\sigma(1)}} \ldots x_{i_{n} i_{\sigma(n)}} .
$$

More generally, the sum of the diagonal immanants of type $\lambda$ gives

$$
j\left(s_{\lambda}\right)=\sum_{i_{1}<\cdots<i_{n}} \sum_{\sigma \in \mathfrak{S}_{n}} \chi^{\lambda}(\sigma) x_{i_{1} i_{\sigma(1)}} \ldots x_{i_{n} i_{\sigma(n)}} .
$$


3.5.4. Involutions. Finally, one can check that the $M_{\sigma}$ with $\sigma$ involutive span a Hopf subalgebra of $\mathfrak{S}$ QSym. Since the number of involutions of $\mathfrak{S}_{n}$ is equal to the number of standard Young tableaux of size $n$, this algebra can be regarded as a commutative version of the Poirier-Reutenauer algebra [27] denoted by FSym and realized in [8]. Notice that this version is also isomorphic to the image of $\mathfrak{S}$ QSYm in the quotient of $R / \mathcal{J}$ by the relations

$$
x_{i j} x_{j k}=0 \text {, for all } i \neq k \text {. }
$$

This construction generalizes to the algebras built on permutations of arbitrary given order.

3.6. Quotients of WSym. Since we have built a subalgebra of $\Pi Q S y m$ isomorphic to QSym, we can define an embedding

$$
i: Q S y m \hookrightarrow \Pi Q S y m=\mathbf{W S y m}^{*},
$$

so that, dually, there is a Hopf epimorphism $i^{*}: \mathbf{W S y m} \rightarrow$ Sym.

The dual basis $V^{I}$ of the $U_{I}$ defined in Equation 60 can be identified with equivalence classes of $\mathbf{S}^{\sigma}$ under the relation

$$
\mathbf{S}^{\sigma} \simeq \mathbf{S}^{\sigma^{\prime}} \text { iff } K(\sigma)=K\left(\sigma^{\prime}\right)
$$

The $\mathbf{S}^{\sigma}$ with $\sigma$ a full cycle are primitive, so that we can take for $V_{n}$ any sequence of primitive generators of Sym.

It turns out that there is another natural epimorphism from WSym to Sym. Using the canonical ordering of set partitions introduced in Section 3.5, that is, the lexicographic ordering on the nondecreasing representatives of the blocks, we can as above associate a composition $K(\pi)$ with $\pi$ and define an equivalence relation

$$
\pi \sim \pi^{\prime} \text { iff } K(\pi)=K\left(\pi^{\prime}\right)
$$

Then, the ideal $\mathfrak{I}$ of WSym generated by the differences

$$
\mathbf{M}_{\pi}-\mathbf{M}_{\pi^{\prime}}, \quad \pi \sim \pi^{\prime}
$$

is a Hopf ideal, and the quotient

$$
\text { WSym/I }
$$

is isomorphic to Sym. The images $V_{I}$ of the $\mathbf{M}_{\pi}$ by the canonical projection are analogs of the monomial symmetric functions in Sym. Indeed, the commutative image $v_{\lambda}$ of $\mathbf{M}_{\pi}$ is proportional to a monomial function:

$$
v_{\lambda}=\prod_{i} m_{i}(\lambda) ! m_{\lambda} .
$$

If we introduce the coefficients $c_{\lambda}$ by

$$
v_{1}^{n}=\sum_{\lambda \vdash n} c_{\lambda} v_{\lambda}
$$

then, the multivariate polynomials

$$
B_{n}\left(x_{1}, \ldots, x_{n}\right)=\sum_{\lambda \vdash n} c_{\lambda} x_{\lambda},
$$


where $x_{\lambda}:=x_{\lambda_{1}} \cdots x_{\lambda_{n}}$, are the exponential Bell polynomials defined by

$$
\sum_{n \geq 0} \frac{t^{n}}{n !} B_{n}\left(x_{1}, \ldots, x_{n}\right)=e^{\sum_{n \geq 1} \frac{x_{n}}{n !} t^{n}} .
$$

3.7. The stalactic monoid. The constructions of Section 3.6 can be interpreted in terms of a kind of Robinson-Schensted correspondence and of a plactic-like monoid. The stalactic congruence is the congruence $\equiv$ on $A^{*}$ generated by the relations

$$
a w a \equiv a a w
$$

for all $a \in A$ and $w \in A^{*}$.

Each stalactic class has a unique representative, its canonical representative of the form

$$
a_{1}^{m_{1}} a_{2}^{m_{2}} \ldots a_{r}^{m_{r}}
$$

with $a_{i} \neq a_{j}$ for $i \neq j$.

We can represent such a canonical word by a tableau-like planar diagram, e.g.,

$$
c^{3} a d^{3} b^{2} \longleftrightarrow \begin{array}{|l|l|l|l|}
\hline c & a & d & b \\
\hline c & & d & b \\
\cline { 1 - 1 } & & \\
& &
\end{array}
$$

Now, there is an obvious algorithm, which consists in scanning a word from left to right and arranging its identical letters in columns, creating a new column to the right when one scans a letter for the first time. Let us call $P(w)$ the resulting canonical word, or, equivalently, its planar representation. We can compute $P(w)$ along with a $Q$-symbol recording the intermediate shapes of the $P$-symbol. For example, to insert $w=c a b c c d b d d$, we have the steps

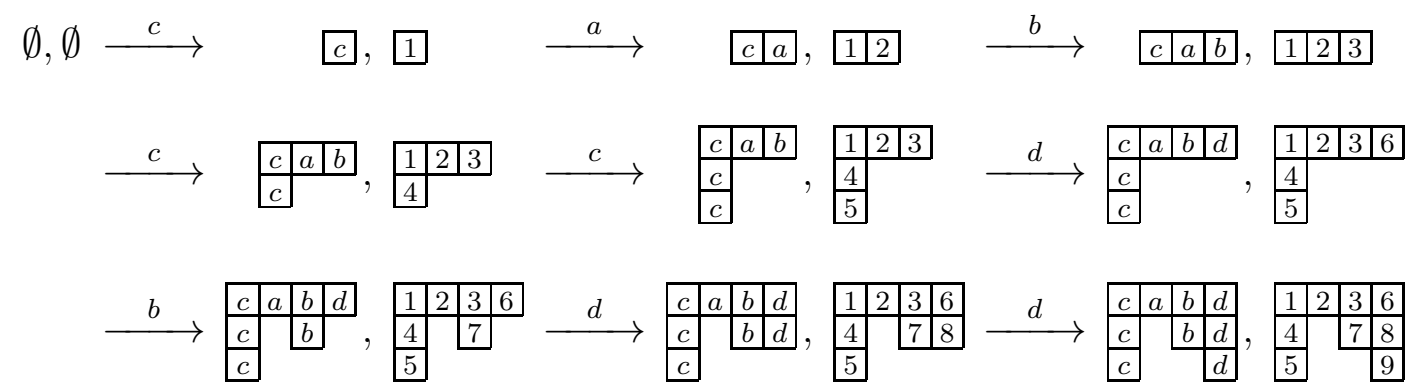

Clearly, the $Q$-symbol can be interpreted as a set partition of $[n]$, whose blocks are the columns. In our example,

$$
Q(c a b c c d b d)=\{\{1,4,5\},\{2\},\{3,7\},\{6,8,9\}\} .
$$

We now see that the natural basis $\mathbf{M}_{\pi}$ of WSym can be characterized as

$$
\mathbf{M}_{\pi}=\sum_{Q(w)=\pi} w
$$

This is completely similar to the definition of the bases $R_{I}$ of $\mathbf{S y m}$ via the hypoplactic congruence, of $\mathbf{S}_{t}$ of FSym via the plactic congruence, and of $\mathbf{P}_{T}$ of PBT via the sylvester congruence [16, 8, 13. 
Note that the defining relation of the stalactic monoid can be presented in a placticlike way as

$$
a u b a \equiv a u a b
$$

for all $a, b \in A$ and $u \in A^{*}$.

3.8. Other Hopf algebras derived from the stalactic congruence. It is always interesting to investigate the behaviour of certain special classes of words under analogs of the Robinson-Schensted correspondence. This section presents a few examples leading to interesting combinatorial sequences.

3.8.1. Parking functions. Recall that a word on $A=\{1, \ldots\}$ is a parking function if its nondecreasing reordering $u_{1} \cdots u_{k}$ satisfies $u_{i} \leq i$. The number of stalactic classes of parking functions of size $n$ can be combinatorially determined as follows.

Since the congruence does not change the evaluation of a word, and since if a word is a parking function, then all its permutations are, one can restrict to a rearrangement class, containing a unique nondecreasing parking function. Those are known to be counted by Catalan numbers. Now, the rearrangement class of a nondecreasing parking function $p$ has exactly $l$ ! congruence classes if $l$ is the number of different letters of $p$.

The counting of nondecreasing parking functions $p$ by their number of different letters is obviously the same as the counting of Dyck paths by their number of peaks, given by the Narayana triangle (sequence A001263 in [31]). To get the number of stalactic classes of parking functions, one has to multiply the $i$ th column by $i$, , that is the definition of the unsigned Lah numbers (sequence A089231 in [31]), which count with the additional parameter of number of lists, the number of sets of lists (sequence A000262 in [31]), that is, the number of set partitions of $[n]$ with an ordering inside each block but no order among the blocks. The first values are

$$
1,3,13,73,501,4051,37633,394353,4596553, \ldots
$$

whereas the first rows of the Narayana and unsigned Lah triangles are

$\begin{array}{cccccccccccc}1 & & & & & & 1 & & & & & \\ 1 & 1 & & & & & 1 & 2 & & & & \\ 1 & 3 & 1 & & & & 1 & 6 & 6 & & & \\ 1 & 6 & 6 & 1 & & & 1 & 12 & 36 & 24 & & \\ 1 & 10 & 20 & 10 & 1 & & 1 & 20 & 120 & 240 & 120 & \\ 1 & 15 & 50 & 50 & 15 & 1 & 1 & 30 & 300 & 1200 & 1800 & 720\end{array}$

Figure 1. The Narayana and unsigned Lah triangles.

One can also obtain this last result by pure algebraic calculations as follows. The Frobenius characteristic of the representation of $\mathfrak{S}_{n}$ on $\mathrm{PF}_{n}$ is

$$
\operatorname{ch}\left(\mathrm{PF}_{n}\right)=\frac{1}{n+1} h_{n}((n+1) X)=\frac{1}{n+1} \sum_{\mu \vdash n} m_{\mu}(n+1) h_{\mu}(X) .
$$


In this expression, each $h_{\mu}(X)$ is the characteristic of the permutation representation on a rearrangement class of words, with $\mu_{1}$ occurrences of some letter $i_{1}, \mu_{2}$ of some other letter $i_{2}$, and so on. Hence, the number of stalactic classes in each such rearrangement class is $l(\mu)$ !, and the total number of stalactic classes of parking functions is

$$
a_{n}=\frac{1}{n+1} \sum_{\mu \vdash n} m_{\mu}(n+1) l(\mu) !
$$

Since $g(z)=\sum_{n \geq 0} z^{n} \operatorname{ch}\left(\mathrm{PF}_{n}\right)$ solves the functional equation [26]

$$
g(z)=\sum_{n \geq 0} z^{n} h_{n}(X) g(z)^{n}
$$

we see that the exponential generating function is

$$
A(z)=\sum_{n \geq 0} \alpha_{n} \frac{z^{n}}{n !}=\int_{0}^{\infty} e^{-x} g(z) d x,
$$

in the special case where $h_{n}=x$ for all $n \geq 1$, that is

$$
g(z)=1+x \frac{z g(z)}{1-z g(z)}
$$

so that

$$
A(z)=\exp \left(\frac{z}{1-z}\right) \text {. }
$$

Hence, $\alpha_{n}$ is the number of 'sets of lists', giving back sequence A000262 of [31]. It would be interesting to find a natural bijection between the stalactic classes of parking functions and sets of lists, compatible with the algebraic structures.

Now, recall that the Hopf algebra of parking functions PQSym* is spanned by polynomials

$$
\mathbf{G}_{\mathbf{a}}(A):=\sum_{\operatorname{Park}(w)=\mathbf{a}} w
$$

As usual, it is easy to show that the equivalence defined by

$$
G_{\mathbf{a}^{\prime}}(A) \equiv \mathbf{G}_{\mathbf{a}^{\prime \prime}}(A)
$$

iff $\mathbf{a}^{\prime}$ and $\mathbf{a}^{\prime \prime}$ are stalactically congruent, is such that the quotient $\mathbf{P Q S y m} \mathbf{S m}^{*} / \equiv$ is a Hopf algebra.

3.8.2. Endofunctions. The same methods allow to see that the number $\beta_{n}$ of stalactic classes of endofunctions on $n$ letters is given by

$$
\beta_{n}=\sum_{k=0}^{n}\left(\begin{array}{l}
n-1 \\
k-1
\end{array}\right)\left(\begin{array}{l}
n \\
k
\end{array}\right) k !
$$

It is sequence A052852 of [31, whose first terms are

$$
1,4,21,136,1045,9276,93289,1047376,12975561,175721140, \ldots
$$


and whose exponential generating series is

$$
\frac{z}{1-z} e^{\frac{z}{1-z}}
$$

As before, the classes counted by those numbers can be counted with the additionnal parameter given by the number of different letters of their representatives, so that one gets a new triangle, the Endt triangle. Moreover, this triangle is obtained by multiplying column $i$ by $i$ ! from a more classical triangle, counting the integer compositions of $2 n$ in $n$ parts with a given number of ones, the Tw triangle. Here are the first rows of both triangles:

$\begin{array}{cccccccccccc}1 & & & & & & 1 & & & & & \\ 2 & 1 & & & & & 2 & 2 & & & & \\ 3 & 6 & 1 & & & & 3 & 12 & 6 & & & \\ 4 & 18 & 12 & 1 & & & 4 & 36 & 72 & 24 & & \\ 5 & 40 & 60 & 20 & 1 & & 5 & 80 & 360 & 380 & 120 & \\ 6 & 75 & 200 & 150 & 30 & 1 & 6 & 150 & 1200 & 3600 & 3600 & 720\end{array}$

Figure 2. The Tw and Endt triangles.

From the algebraic point of view, the Frobenius characteristic of $[n]^{n}$ is

$$
\operatorname{ch}\left([n]^{n}\right)=h_{n}(n X)=\sum_{\mu \vdash n} m_{\mu}(n) h_{\mu}(X),
$$

so that $\beta_{n}=\sum_{\mu \vdash n} m_{\mu}(n) l(\mu)$ !, and the same method gives directly the exponential generating series of $\beta_{n}$.

As above, the quotient of EQSym by $\mathbf{S}^{f^{\prime}} \equiv \mathbf{S}^{f^{\prime \prime}}$ iff $f^{\prime}$ and $f^{\prime \prime}$ are stalactically congruent is a Hopf algebra.

3.8.3. Initial words. Recall that initial words are words on the alphabet of integers so that, if letter $n$ appears in $w$, then $n-1$ also appears. The same method allows to see that the number $\gamma_{n}$ of stalactic classes of initial words on $n$ letters is

$$
\gamma_{n}=\sum_{k=0}^{n}\left(\begin{array}{l}
n-1 \\
k-1
\end{array}\right) k !
$$

It is sequence A001339 of 31, whose first terms are

$$
1,3,11,49,261,1631,11743,95901,876809,8877691,98641011, \ldots
$$

and whose exponential generating series is

$$
\frac{e^{z}}{(1-z)^{2}}
$$

As before, the classes counted by those numbers can be counted with the additionnal parameter given by the number of different letters of their representatives, so that one gets a new triangle, the Arr triangle. Moreover, this triangle is obtained by 


\begin{tabular}{|c|c|c|c|c|c|c|c|c|}
\hline 1 & & & & 1 & & & & \\
\hline 1 & & & & 1 & 2 & & & \\
\hline 2 & 1 & & & 1 & 4 & 6 & & \\
\hline 3 & 3 & 1 & & 1 & 6 & 18 & 24 & \\
\hline 4 & 6 & 4 & 1 & 1 & 8 & 36 & 96 & 120 \\
\hline 5 & 10 & 10 & $\begin{array}{ll}5 & 1\end{array}$ & 1 & 10 & 60 & 240 & 600 \\
\hline
\end{tabular}

Figure 3. The Pascal and Arr triangles.

multiplying column $i$ by $i$ ! in the Pascal triangle. Here are the first rows of both triangles:

Again, the stalactic quotient of WQSym [24, 12] is a Hopf algebra.

3.8.4. Generic case. Let $A$ be an alphabet, and $V=\mathbb{C} A$. The generic symmetric function

$$
f_{n}=\sum_{\mu \vdash n} l(\mu) ! m_{\mu}(X)
$$

is the character of $G L(V)$ in the image of $V^{\otimes n}$ in the quotient of $T(V) \sim \mathbb{K}\langle A\rangle$ by the stalactic congruence. It is Schur-positive, and can be explicitly expanded on the Schur basis. Indeed, one has

$$
f_{n}=\sum_{k=0}^{n} c_{k} s_{(n-k, k)} .
$$

The coefficients $c_{k}$ are given by sequence A000255 of [31], whose first terms are

$$
1,1,3,11,53,309,2119,16687,148329,1468457,16019531, \ldots
$$

To see this, write again

$$
l(\mu) !=\int_{0}^{\infty} t^{l(\mu)} e^{-t} d t .
$$

Then

$$
f=\sum_{n \geq 0} f_{n}=\int_{0}^{\infty} e^{-t} \sum_{\mu} t^{l(\mu)} m_{\mu} d t .
$$

and

$$
\begin{aligned}
\sum_{\mu} t^{l(\mu)} m_{\mu}=\prod_{i \geq 1}\left(1+\frac{t x_{i}}{1-x_{i}}\right) & =\prod_{i \geq 1} \frac{1-(1-t) x_{i}}{1-x_{i}} \\
& =\left(\sum_{k \geq 0}(t-1)^{k} e_{k}(X)\right)\left(\sum_{l \geq 0} h_{l}(X)\right) .
\end{aligned}
$$

Since

$$
\int_{0}^{\infty} e^{-t}(t-1)^{k} d t=d_{k}
$$


the number of derangements in $\mathfrak{S}_{n}$, we have finally

$$
f=\sum_{n \geq 0} \sum_{k=0}^{n} d_{k} e_{k}(X) h_{n-k}(X) .
$$

Expanding

$$
e_{k} h_{n-k}=s_{\left(n-k, 1^{k}\right)}+s_{n-k+1,1^{k-1}},
$$

we get Formula (103). Alternatively, we can express $c_{k}$ as

$$
c_{k}=\int_{0}^{\infty} e^{-t} t(t-1)^{k} d t
$$

since the term of degree $n$ in Equation (107) is

$$
t \sum_{k=0}^{n}(t-1)^{k} s_{\left(n-k, 1^{k}\right)} .
$$

The exponential generating series of these numbers is given by

$$
\frac{e^{-z}}{(1-z)^{2}}
$$

\section{Structure OF SSym}

4.1. A realization of $\mathfrak{S S y m}$. In the previous section, we have built a commutative algebra of permutations $\mathfrak{S}$ QSym from explicit polynomials on a set of auxiliary variables $x_{i j}$. One may ask whether its non-commutative dual admits a similar realization in terms of non-commuting variables $a_{i j}$.

We shall find such a realization, in a somewhat indirect way, by first building from scratch a Hopf algebra of permutations $\mathbf{\Phi S y m ~} \subset \mathbb{K}\left\langle a_{i j} \mid i, j \geq 1\right\rangle$, whose operations can be described in terms of the cycle structure of permutations. Its coproduct turns out to be cocommutative, and the isomorphism with $\mathfrak{S S y m}$ follows as above from the Cartier-Milnor-Moore theorem.

Let $\left\{a_{i j}, i, j \geq 1\right\}$ be an infinite set of non-commuting indeterminates. We use the biword notation

$$
a_{i j} \equiv\left[\begin{array}{l}
i \\
j
\end{array}\right], \quad\left[\begin{array}{l}
i_{1} \\
j_{1}
\end{array}\right] \cdots\left[\begin{array}{l}
i_{n} \\
j_{n}
\end{array}\right] \equiv\left[\begin{array}{l}
i_{1} \ldots i_{n} \\
j_{1} \ldots j_{n}
\end{array}\right]
$$

Let $\sigma \in \mathfrak{S}_{n}$ and let $\left(c_{1}, \ldots, c_{k}\right)$ be a decomposition of $\sigma$ into disjoint cycles. With any cycle, one associates its cycle words, that is, the words obtained by reading the successive images of any element of the cycle. For example, the cycle words associated with the cycle $(1342)$ are $1342,2134,3421,4213$. For all word $w$, we denote by $C(w)$ the cycle associated with the inverse of its standardized word.

We now define

$$
\phi_{\sigma}:=\sum_{x, a}\left[\begin{array}{l}
x \\
a
\end{array}\right],
$$

where the sum runs over all words $x$ such that $x_{i}=x_{j}$ iff $i$ and $j$ belong to the same cycle of $\sigma$, and such that the standardized word of the subword $x$ of $a$ whose letter 
positions belong to cycle $c_{l}$ satisfied $C(x)=c_{l}$. By extension, such biwords will be said to have $\sigma$ as cycle decomposition.

Note that any biword appears in the expansion of exactly one $\phi_{\sigma}$.

Example 4.1.

$$
\begin{gathered}
\phi_{12}=\sum_{x \neq y}\left[\begin{array}{ll}
x & y \\
a & b
\end{array}\right] . \\
\sum_{41352=}\left[\begin{array}{lllll}
x & x & y & x & x \\
a & b & c & d & e
\end{array}\right] .
\end{gathered}
$$

Theorem 4.2. The $\phi_{\sigma}$ span a subalgebra $\mathbf{\Phi S y m ~ o f ~} \mathbb{K}\left\langle a_{i j} \mid i, j \geq 1\right\rangle$. More precisely,

$$
\phi_{\alpha} \phi_{\beta}=\sum g_{\alpha, \beta}^{\sigma} \phi_{\sigma}
$$

where $g_{\alpha, \beta}^{\sigma} \in\{0,1\}$. Moreover, $\mathbf{\Phi S y m}$ is free over the set

$$
\left\{\phi_{\alpha} \mid \alpha \text { connected }\right\} \text {. }
$$

Proof - Let $\sigma^{\prime}$ and $\sigma^{\prime \prime}$ be two permutations and let $w$ be a biword appearing in $\phi_{\sigma^{\prime}} \phi_{\sigma^{\prime \prime}}$. The multiplicity of $w$ is 1 . To get the first part of the theorem, we only need to prove that all words $w^{\prime}$ appearing in the same $\phi_{\sigma}$ as $w$ also appear in this product. Given a letter $x$ appearing in the first row of $w$ at some positions, the subwords $a$ of the elements of $\phi_{\sigma}$ taken from the second row at those positions, have the same image by $C$ as the corresponding element of $w$.

Thus, we onyl have to prove that all words $w$ having the same image by $C$ satisfy that all their prefixes (and suffixes) of a given length have also same image by $C$. It is sufficient to prove the result on permutations. Now, given two permutations $\sigma$ and $\tau, \sigma^{-1}$ and $\tau^{-1}$ are cycle words of the same cycle, iff for some $k, \tau=\gamma^{k} \sigma$ where $\gamma$ is the cyclic permutation $(12 \cdots n)$. Since is also the case for the standardized words of the prefixes of both permutations of a given length, the property holds.

Moreover, any biword can be uniquely written as a concatenation of a maximal number of biwords such that no letter appears in the first row of two different biwords and that the letters of the second row of a biword are all smaller than the letters of the second row of the next one. This proves that the $\phi_{\alpha}$ where $\alpha$ is connected are free. The usual generating series argument then proves that those elements generate ФSym.

To give the precise expression of the product $\phi_{\alpha} \phi_{\beta}$, we first need to define two operations on cycles.

The first operation is just the circular shuffle on disjoint cycles: if $c_{1}^{\prime}$ and $c_{1}^{\prime \prime}$ are two disjoint cycles, their cyclic shuffle $c_{1}^{\prime} \omega c_{1}^{\prime \prime}$ is the set of cycles $c_{1}$ such that their cycle words are obtained by applying the usual shuffle on the cycle words of $c_{1}^{\prime}$ and $c_{1}^{\prime \prime}$. This definition makes sense because a shuffle of cycle words associated with two words on disjoint alphabets splits as a union of cyclic classes. 
For example, the cyclic shuffle (132)ш(45) gives the set of cycles

$$
\begin{aligned}
& \{(13245),(13425),(13452),(14325),(14352),(14532), \\
& (13254),(13524),(13542),(15324),(15342),(15432)\} .
\end{aligned}
$$

These cycles correspond to the following list of permutations which are those appearing in Equation (127), except for the first one which will be found later:

$$
\begin{array}{r}
\{34251,35421,31452,45231,41532,41253, \\
35214,34512,31524,54213,51423,51234\} .
\end{array}
$$

Let us now define an operation on two sets $C_{1}$ and $C_{2}$ of disjoint cycles. We call matching a list of all those cycles, some of the cycles being paired, always one of $C_{1}$ with one of $C_{2}$. The cycles remaining alone are considered to be associated with the empty cycle. With all matchings associate the set of sets of cycles obtained by the cyclic product $\Psi$ of any pair of cycles. The union of those sets of cycles is denoted by $C_{1} \smile C_{2}$.

For example, the matchings corresponding to $C_{1}=\{(1),(2)\}$ and $C_{2}=\{(3),(4)\}$ are:

$$
\begin{aligned}
& \{(1)\}\{(2)\}\{(3)\}\{(4)\},\{(1)\}\{(2),(3)\}\{(4)\},\{(1)\}\{(2),(4)\}\{(3)\}, \\
& \{(1),(3)\}\{(2)\}\{(4)\},\{(1),(3)\}\{(2),(4)\},\{(1),(4)\}\{(2)\}\{(3)\},\{(1),(4)\}\{(2),(3)\},
\end{aligned}
$$

and the product $C_{1} \smile C_{2}$ is then

$$
\begin{aligned}
& \{(1),(2),(3),(4)\},\{(1),(23),(4)\},\{(1),(24),(3)\}, \\
& \{(13),(2),(4)\},\{(13),(24)\},\{(14),(2),(3)\}, \quad\{(14),(23)\} .
\end{aligned}
$$

Remark that this calculation is identical with the Wick formula in quantum field theory (see [6] for an explanation of this coincidence).

We are now in a position to describe the product $\phi_{\sigma} \phi_{\tau}$ :

Proposition 4.3. let $C_{1}$ be the cycle decomposition of $\sigma$ and $C_{2}$ be the cycle decomposition of $\tau$, shifted by the size of $\sigma$. Then the permutations indexing the elements appearing in the product $\phi_{\sigma} \phi_{\tau}$ are the permutations whose cycle decompositions belong to $C_{1} \smile C_{2}$.

Proof - Recall that the product $\phi_{\sigma} \phi_{\tau}$ is a sum of biwords with multiplicity one since a word appears in exactly one $\phi_{\sigma}$. So we only have to prove that biwords appearing in $\phi_{\sigma} \phi_{\tau}$ are the same as biwords whose cycle decomposition are in $C_{1} \smile C_{2}$. First, by definition of the cyclic shuffle and of operation $\smile$, if a biword has its cycle decomposition in $C_{1} \smile C_{2}$, its prefix of size $n$ has cycle decomposition $C_{1}$ whereas its suffix of size $p$ has cycle decomposition $C_{2}$, where $n$ (resp. $p$ ) is the size of $C_{1}$ (resp. $\left.C_{2}\right)$.

Conversely, let $w_{1}$ (resp. $w_{2}$ ) be a biword with cycle decomposition $C_{1}$ (resp. $C_{2}$ ) and let us consider $w=w_{1} \cdot w_{2}$. For all letters in the first row of $w$, either it only appears in $w_{1}$, either only in $w_{2}$, or in both $w_{1}$ and $w_{2}$. In the first two cases, we obtain the corresponding cycle of $C_{1}$ (or $C_{2}$, shifted). In the last case, the cycle decomposition of the word of the second row corresponding to this letter belongs to 
the cyclic shuffle of the corresponding cycles of $C_{1}$ and $C_{2}$ (hence matching those two cycles). Indeed, if $\operatorname{Std}^{-1}\left(w_{1}\right)$ is a cycle word of $c_{1}$ and $\operatorname{Std}^{-1}\left(w_{2}\right)$ is a cycle word of $c_{2}$, then $\operatorname{Std}^{-1}(w)$ is a cycle word of an element of the cyclic shuffle $c_{1} \uplus c_{2}$ : it is already known when $\operatorname{Std}^{-1}\left(w_{1}\right)=c_{1}$ and $\operatorname{Std}^{-1}\left(w_{2}\right)=c_{2}$ since it is the definition of FQSym [8] and cyclying $c_{1}$ or $c_{2}$ only amounts to cycle $c_{2}$, hence copying the definition of the cyclic shuffle.

For example, with $\sigma=\tau=12$, one finds that $C_{1}=\{(1),(2)\}$ and $C_{2}=\{(3),(4)\}$. It is then easy to check that one goes from Equation (123) to Equation (125) by computing the corresponding permutations.

Example 4.4.

$$
\begin{gathered}
\phi_{12} \phi_{21}=\phi_{1243}+\phi_{1342}+\phi_{1423}+\phi_{3241}+\phi_{4213} . \\
\phi_{12} \phi_{12}=\phi_{1234}+\phi_{1324}+\phi_{1432}+\phi_{3214}+\phi_{3412}+\phi_{4231}+\phi_{4321} . \\
\phi_{1} \phi_{4312}=\phi_{15423}+\phi_{25413}+\phi_{35421}+\phi_{45123}+\phi_{51423} . \\
\phi_{312} \phi_{21}=\phi_{31254}+\phi_{31452}+\phi_{31524}+\phi_{34251}+\phi_{34512}+\phi_{35214}+\phi_{35421} \\
+\phi_{41253}+\phi_{41532}+\phi_{45231}+\phi_{51234}+\phi_{51423}+\phi_{54213} .
\end{gathered}
$$

Let us recall a rather general recipe to obtain the coproduct of a combinatorial Hopf algebra from a realization in terms of words on an ordered alphabet $X$. Assume that $X$ is the ordered sum of two mutually commuting alphabets $X^{\prime}$ and $X^{\prime \prime}$. Then define the coproduct as $\Delta(F)=F\left(X^{\prime}+X^{\prime \prime}\right)$, identifying $F^{\prime} \otimes F^{\prime \prime}$ with $F^{\prime}\left(X^{\prime}\right) F^{\prime \prime}\left(X^{\prime \prime}\right)$ [8, 23.

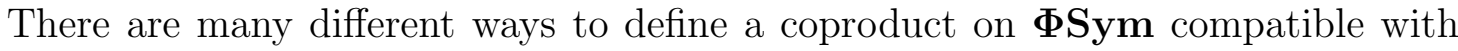
the realization since there are many ways to order an alphabet of biletters: order the letters of the first alphabet, order the letters of the second alphabet, or order lexicographically with respect to one alphabet and then to the second.

In the sequel, we only consider the coproduct obtained by ordering the biletters with respect to the first alphabet so that $\phi_{\sigma}$ is primitive if $\sigma$ consists of only one cycle. More precisely, thanks to the definition of the $\phi$, it is easy to see that it corresponds to the unshuffling of the cycles of a permutation:

$$
\Delta \phi_{\sigma}:=\sum_{(\alpha, \beta)} \phi_{\alpha} \otimes \phi_{\beta}
$$

where the sum is taken over all pairs of permutations $(\alpha, \beta)$ such that $\alpha$ is obtained by renumbering the elements of a subset of cycles of $\sigma$ (preserving the relative order of values), and $\beta$ by doing the same on the complementary subset of cycles. For example, if $\sigma=(1592)(36)(4)(78)$, the subset (1592)(4) gives $\alpha=(1452)(3)$ and $\beta=(12)(34)$. 
Example 4.5.

$$
\begin{gathered}
\Delta \phi_{12}=\phi_{12} \otimes 1+2 \phi_{1} \otimes \phi_{1}+1 \otimes \phi_{12} . \\
\Delta \phi_{312}=\phi_{312} \otimes 1+1 \otimes \phi_{312} .
\end{gathered}
$$

$$
\Delta \phi_{4231}=\phi_{4231} \otimes 1+2 \phi_{321} \otimes \phi_{1}+\phi_{21} \otimes \phi_{12}+\phi_{12} \otimes \phi_{21}+2 \phi_{1} \otimes \phi_{321}+1 \otimes \phi_{4231} .
$$

The following theorem is a direct consequence of the definition of the coproduct on the realization.

Theorem 4.6. $\Delta$ is an algebra morphism, so that $\mathbf{\Phi S y m}$ is a graded bialgebra (for the grading $\operatorname{deg} \phi_{\sigma}=n$ if $\left.\sigma \in \mathfrak{S}_{n}\right)$. Moreover, $\Delta$ is cocommutative.

The same reasoning as in Section 3 shows that

Theorem 4.7. SSym and $\mathbf{\Phi S y m}$ are isomorphic as Hopf algebras.

To get the explicit isomorphism sending $\phi$ to $\mathbf{S}$, let us first recall that a connected permutation is a permutation $\sigma$ such that $\sigma([1, k]) \neq[1, k]$ for any $k \in[1, n-1]$. Any permutation $\sigma$ has a unique maximal factorization $\sigma=\sigma_{1} \bullet \cdots \bullet \sigma_{r}$ into connected permutations. We then define

$$
\mathbf{S}_{\sigma}^{\prime}:=\phi_{\sigma_{1}} \cdots \phi_{\sigma_{r}}
$$

Then

$$
\mathbf{S}_{\sigma}^{\prime}=\phi_{\sigma_{1} \bullet \cdots \bullet \sigma_{r}}+\sum_{\mu} \phi_{\mu}
$$

where all permutations $\mu$ have strictly less cycles than $r$. So the $\mathbf{S}^{\prime}$ form a basis of $\mathbf{\Phi S y m}$. Moreover, they are a multiplicative basis with product given by shifted concatenation of permutations, so that they multiply as the $\mathbf{S}$ do. Moreover, the coproduct of $\mathbf{S}_{\sigma}^{\prime}$ is the same as for $\phi_{\sigma}$, so the same as for $\mathbf{S}^{\sigma}$. So both bases $\mathbf{S}$ and $\mathbf{S}^{\prime}$ have the same product and the same coproduct. This proves

Proposition 4.8. The linear map $\mathbf{S}^{\sigma} \mapsto \mathbf{S}_{\sigma}^{\prime}$ realizes the Hopf isomorphism between SSym and $\mathbf{\Phi}$ Sym.

There is another natural isomorphism: define

$$
\mathbf{S}_{\sigma}^{\prime \prime}:=\sum_{x, a}\left[\begin{array}{l}
x \\
a
\end{array}\right],
$$

where the sum runs over all words $x$ such that $x_{i}=x_{j}$ if (but not only if) $i$ and $j$ belong the same cycle of $\sigma$ and such that the standardized word of the subword of $a$ consisting of the indices of cycle $c_{l}$ is equal to the inverse of the standardized word of a cycle word of $c_{l}$. 
That both bases $\mathbf{S}^{\prime}$ and $\mathbf{S}^{\prime \prime}$ have the same product and coproduct comes from the fact that if $\left(c_{1}\right) \cdots\left(c_{p}\right)$ is the cycle decomposition of $\sigma$,

$$
\mathbf{S}_{\sigma}^{\prime \prime}=\sum_{(c) ;(c) \in\left(c_{1}\right) \smile\left(c_{2}\right) \smile \cdots \smile\left(c_{p}\right)} \phi_{(c)} .
$$

For example,

$$
\begin{aligned}
\mathbf{S}_{2431}^{\prime \prime}=\mathbf{S}_{(124)(3)}^{\prime \prime} & =\phi_{(124)(3)}+\phi_{(1423)}+\phi_{(1234)}+\phi_{(1324)} \\
& =\phi_{2431}+\phi_{4312}+\phi_{2341}+\phi_{3421} .
\end{aligned}
$$

4.2. Quotients of $\mathbf{\Phi S y m}$. Let $I$ be the ideal of $\mathbf{\Phi S y m}$ generated by the differences

$$
\phi_{\sigma}-\phi_{\tau}
$$

where $\sigma$ and $\tau$ have the same cycle type.

The definitions of its product and coproduct directly imply that $I$ is a Hopf ideal. Since the cycle types are parametrized by integer partitions, the quotient $\mathbf{\Phi S \mathbf { S m } / I}$ has a basis $Y_{\lambda}$, corresponding to the class of $\phi_{\sigma}$, where $\sigma$ has $\lambda$ as cycle type.

From Equations (124)-(127), one finds:

\section{Example 4.9.}

$$
\begin{gathered}
Y_{11} Y_{2}=Y_{211}+4 Y_{31}, \quad Y_{11}^{2}=Y_{1111}+2 Y_{22}+4 Y_{211} \\
Y_{1} Y_{4}=Y_{41}+4 Y_{5}, \quad Y_{3} Y_{2}=Y_{32}+12 Y_{5} .
\end{gathered}
$$

Theorem 4.10. $\mathbf{\Phi S y m} / I$ is isomorphic to Sym, the Hopf algebra of ordinary symmetric functions,

If one writes $\lambda=\left(\lambda_{1}, \ldots, \lambda_{p}\right)=\left(1^{m_{1}}, \ldots, k^{m_{k}}\right)$, an explicit isomorphism is given by

$$
Y_{\lambda} \mapsto \frac{\prod_{i=1}^{k} m_{i} !}{\prod_{j=1}^{p}\left(\lambda_{j}-1\right) !} m_{\lambda} .
$$

Proof - This follows from the description of $\phi_{\sigma} \phi_{\tau}$ given in Proposition 4.3.

\section{PARKing FUnCtions AND TREes}

5.1. A commutative algebra of parking functions. It is also possible to build a commutative pendant of the Hopf algebra of parking functions introduced in [22]: let $\mathrm{PF}_{n}$ be the set of parking functions of length $n$. For $\mathbf{a} \in \mathrm{PF}_{n}$, set, as before

$$
M_{\mathbf{a}}:=\sum_{i_{1}<\cdots<i_{n}} x_{i_{1} i_{\mathbf{a}(1)}} \cdots x_{i_{n} i_{\mathbf{a}(n)}} .
$$

Then, using once more the same arguments of Section 2, we conclude that the $M_{\mathbf{a}}$ form a linear basis of a $\mathbb{Z}$-subalgebra PQSym of EQSym, which is also a sub-coalgebra if one defines the coproduct in the usual way. 


\section{Example 5.1.}

$$
\begin{gathered}
M_{1} M_{11}=M_{122}+M_{121}+M_{113} . \\
M_{1} M_{221}=M_{1332}+M_{3231}+M_{2231}+M_{2214} . \\
M_{12} M_{21}=M_{1243}+M_{1432}+M_{4231}+M_{1324}+M_{3214}+M_{2134} . \\
\Delta M_{525124}=M_{525124} \otimes 1+1 \otimes M_{525124} . \\
\Delta M_{4131166}=M_{4131166} \otimes 1+M_{41311} \otimes M_{11}+1 \otimes M_{4131166} .
\end{gathered}
$$

The main interest of the non-commutative and non-cocommutative Hopf algebra of parking functions defined in [22] is that it leads to two algebras of trees. The authors obtain a cocommutative Hopf algebra of planar binary trees by summing over the distinct permutations of parking functions, and an algebra of planar trees by summing over hypoplactic classes.

We shall now investigate whether similar constructions can be found for the commutative version $P Q S y m$.

5.2. From labelled to unlabelled parking graphs. A first construction, which can always be done for Hopf algebras of labelled graphs is to build a subalgebra by summing over labellings. Notice that this subalgebra is the same as the subalgebra obtained by summing endofunctions graphs over their labellings. Those graphs are also known as endofunctions (hence considered there as unlabelled graphs) in [3].

The dimension of this subalgebra in degree $n$ is equal to the number of unlabelled parking graphs

$$
1,1,3,7,19,47,130,343,951,2615,7318,20491,57903,163898, \ldots
$$

known as sequence A001372 in [31]. For example, here are the 7 unlabelled parking graphs of size 3 (to be compared with the 16 parking functions):

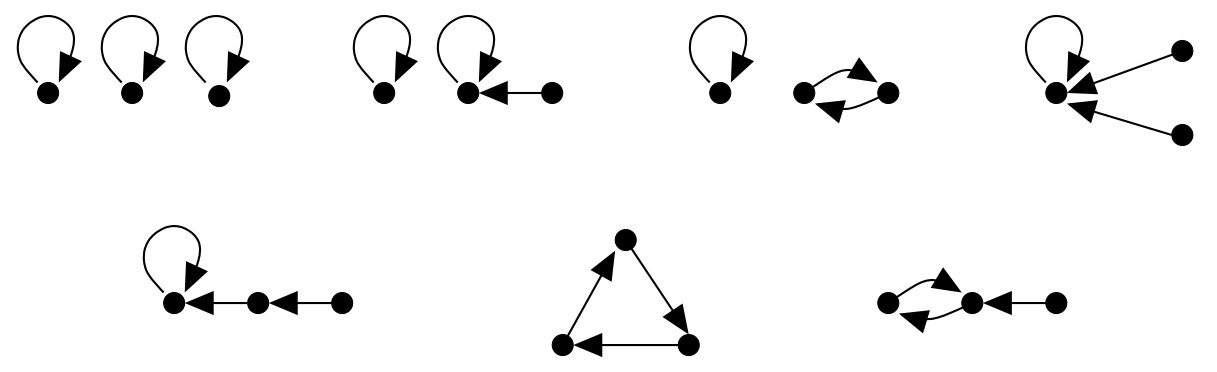

The product of two such unlabelled graphs is the concatenation of graphs and the coproduct of an unlabelled graph is the unshuffle of its connected subgraphs. So this algebra is isomorphic to the polynomial algebra on generators indexed by connected parking graphs. 
5.3. Binary trees and nondecreasing parking functions. One can easily check that in PQSym, summing over parking functions having the same reordering does not lead to a subalgebra. However, if we denote by $I$ the subspace of PQSym spanned by the $M_{\mathbf{a}}$ where $\mathbf{a}$ is not nondecreasing, $I$ is an ideal and a coideal, and CQSym $:=$ PQSym $/ I$ is therefore a commutative Hopf algebra with basis given by the classes $M_{\pi}:=\overline{M_{\mathbf{a}}}$ labelled by nondecreasing parking functions.

Notice that CQSym is also isomorphic to the image of PQSym in the quotient of $R / \mathcal{J}$ by the relations

$$
x_{i j} x_{k l}=0, \text { for all } i<k \text { and } j>l .
$$

The dual basis of $M_{\pi}$ is

$$
S^{\pi}:=\sum_{\mathbf{a}} S^{\mathbf{a}}
$$

where the sum is taken over all permutations of $\pi$.

The dual CQSym* is free over the set $S^{\pi}$, where $\pi$ runs over connected nondecreasing parking functions. So if one denotes by CQSym the Catalan algebra defined in [22], the usual Cartier-Milnor-Moore argument then shows that

$$
\text { CQSym } \sim \text { CQSym* }{ }^{*}, \quad \text { CQSym } \sim \text { CQSym* }^{*}
$$

5.4. From nondecreasing parking functions to rooted forests. Nondecreasing parking functions correspond to parking graphs of a particular type: namely, rooted forests with a particular labelling (it corresponds to nondecreasing maps), the root being given by the loops in each connected component.

Taking sums over the allowed labellings of a given rooted forest, we get that the

$$
M_{F}:=\sum_{\operatorname{supp}(\pi)=F} M_{\pi},
$$

span a commutative Hopf algebra of rooted forests, which is likely to coincide with the quotient of the Connes-Kreimer algebra [7] by its coradical filtration [2].

\section{QUANTUM VERSIONS}

6.1. Quantum quasi-symmetric functions. When several Hopf algebra structures can be defined on the same class of combinatorial objects, it is tempting to try to interpolate between them. This can be done, for example with compositions: the algebra of quantum quasi-symmetric functions $\operatorname{QSym}_{q}$ [33, 8] interpolates between quasi-symmetric functions and non-commutative symmetric functions.

However, the natural structure on QSym $_{q}$ is not exactly that of a Hopf algebra but rather of a twisted Hopf algebra [18].

Recall that the coproduct of $Q \operatorname{Sym}(X)$ amounts to replace $X$ by the ordered sum $X^{\prime} \dot{+} X^{\prime \prime}$ of two isomorphic and mutually commuting alphabets. On the other hand, QSym $_{q}$ can be realized by means of an alphabet of $q$-commuting letters

$$
x_{j} x_{i}=q x_{i} x_{j} \text {, for } j>i .
$$


Hence, if we define a coproduct on $Q S y m_{q}$ by

$$
\Delta_{q} f(X)=f\left(X^{\prime} \dot{+} X^{\prime \prime}\right),
$$

with $X^{\prime}$ and $X^{\prime \prime} q$-commuting with each other, it will be an algebra morphism

$$
\operatorname{QSym}_{q} \rightarrow \operatorname{QSym}_{q}\left(X^{\prime} \dot{+} X^{\prime \prime}\right) \simeq \operatorname{QSym}_{q} \otimes_{\chi} \operatorname{QSym}_{q}
$$

for the twisted product of tensors

$$
(a \otimes b) \cdot\left(a^{\prime} \otimes b^{\prime}\right)=\chi\left(b, a^{\prime}\right)\left(a a^{\prime} \otimes b b^{\prime}\right),
$$

where

$$
\chi\left(b, a^{\prime}\right)=q^{\operatorname{deg}(b) \cdot \operatorname{deg}\left(a^{\prime}\right)}
$$

for homogeneous elements $b$ and $a^{\prime}$.

It is easily checked that $\Delta_{q}$ is actually given by the same formula as the usual coproduct of QSym, that is

$$
\Delta_{q} M_{I}=\sum_{H \cdot K=I} M_{H} \otimes M_{K}
$$

The dual twisted Hopf algebra, denoted by $\mathbf{S y m}$, is isomorphic to $\mathbf{S y m}$ as a algebra. If we denote by $S^{I}$ the dual basis of $M_{I}, S^{I} S^{J}=S^{I \cdot J}$, and $\mathbf{S y m} q$ is freely generated by the $S^{(n)}=S_{n}$, whose coproduct is

$$
\Delta_{q} S_{n}=\sum_{i+j=n} q^{i j} S_{i} \otimes S_{j}
$$

As above, $\Delta_{q}$ is an algebra morphism

$$
\mathbf{S y m}_{q} \rightarrow \mathbf{S y m}_{q} \otimes_{\chi} \mathbf{S y m}_{q}
$$

where $\chi$ is again defined by Equation (156).

6.2. Quantum free quasi-symmetric functions. The previous constructions can be lifted to FQSym. Recall from [8] that $\phi\left(\mathbf{F}_{\sigma}\right)=q^{l(\sigma)} F_{c(\sigma)}$ is an algebra homomorphism FQSym $\rightarrow$ QSym $_{q}$, which is in fact induced by the specialization $\phi\left(a_{i}\right)=x_{i}$ of the underlying free variables $a_{i}$ to $q$-commuting variables $x_{i}$.

The coproduct of FQSym is also defined by

$$
\Delta \mathbf{F}(A)=\mathbf{F}\left(A^{\prime} \dot{+} A^{\prime \prime}\right),
$$

where $A^{\prime}$ and $A^{\prime \prime}$ are two mutually commuting copies of $A$ [8]. If instead one sets $a^{\prime \prime} a^{\prime}=q a^{\prime} a^{\prime \prime}$, one obtains again a twisted Hopf algebra structure $\mathbf{F Q S y m}_{q}$ on FQSym, for which $\phi$ is a homomorphism. With these definitions at hand, one can see that the arguments given in [33] to establish the results recalled in Section 6.1 prove in fact the following more general result:

Theorem 6.1. Let $A^{\prime}$ and $A^{\prime \prime}$ be q-commuting copies of the ordered alphabet $A$, i.e., $a^{\prime \prime} a^{\prime}=q a^{\prime} a^{\prime \prime}$ for $a^{\prime} \in A^{\prime}$ and $a^{\prime \prime} \in A^{\prime \prime}$. Then, the coproduct

$$
\Delta_{q} f=f\left(A^{\prime} \dot{+} A^{\prime \prime}\right)
$$


defines a twisted Hopf algebra structure. It is explictly given in the basis $\mathbf{F}_{\sigma}$ by

$$
\Delta_{q} \mathbf{F}_{\sigma}=\sum_{\alpha \cdot \beta=\sigma} q^{\operatorname{inv}(\alpha, \beta)} \mathbf{F}_{\operatorname{Std}(\alpha)} \otimes \mathbf{F}_{\operatorname{Std}(\beta)}
$$

where $\operatorname{inv}(\alpha, \beta)$ is the number of inversions of $\sigma$ with one element in $\alpha$ and the other in $\beta$.

More precisely, $\Delta_{q}$ is an algebra morphism with values in the twisted tensor product of graded algebras FQSym $\otimes_{\chi}$ FQSym where $(a \otimes b)\left(a^{\prime} \otimes b^{\prime}\right)=\chi\left(b, a^{\prime}\right)\left(a a^{\prime} \otimes b b^{\prime}\right)$ and $\chi\left(b, a^{\prime}\right)=q^{\operatorname{deg}(b) \cdot \operatorname{deg}\left(a^{\prime}\right)}$ for homogeneous elements $b, a^{\prime}$.

The map $\phi: \mathbf{F Q S y m}_{q} \rightarrow$ QSym $_{q}$ defined by

$$
\phi\left(\mathbf{F}_{\sigma}\right)=q^{l(\sigma)} F_{c(\sigma)}
$$

is a morphism of twisted Hopf algebras.

\section{Example 6.2.}

$$
\Delta_{q} \mathbf{F}_{2431}=\mathbf{F}_{2431} \otimes 1+q^{3} \mathbf{F}_{132} \otimes \mathbf{F}_{1}+q^{3} \mathbf{F}_{12} \otimes \mathbf{F}_{21}+q \mathbf{F}_{1} \otimes \mathbf{F}_{321}+1 \otimes \mathbf{F}_{2431} .
$$

$$
\Delta_{q} \mathbf{F}_{3421}=\mathbf{F}_{3421} \otimes 1+q^{3} \mathbf{F}_{231} \otimes \mathbf{F}_{1}+q^{4} \mathbf{F}_{12} \otimes \mathbf{F}_{21}+q^{2} \mathbf{F}_{1} \otimes \mathbf{F}_{321}+1 \otimes \mathbf{F}_{3421}
$$

$$
\begin{gathered}
\Delta_{q} \mathbf{F}_{21}=\mathbf{F}_{21} \otimes 1+q \mathbf{F}_{1} \otimes \mathbf{F}_{1}+1 \otimes \mathbf{F}_{21} . \\
\left(\Delta_{q} \mathbf{F}_{21}\right)\left(\Delta_{q} \mathbf{F}_{1}\right)=\left(\mathbf{F}_{213}+\mathbf{F}_{231}+\mathbf{F}_{321}\right) \otimes 1+\left(\mathbf{F}_{21}+q^{2}\left(\mathbf{F}_{12}+\mathbf{F}_{21}\right)\right) \otimes \mathbf{F}_{1} \\
+\mathbf{F}_{1} \otimes\left(q^{2} \mathbf{F}_{21}+q\left(\mathbf{F}_{12}+\mathbf{F}_{21}\right)\right)+1 \otimes\left(\mathbf{F}_{213}+\mathbf{F}_{231}+\mathbf{F}_{321}\right) . \\
\Delta_{q} \mathbf{F}_{213}=\mathbf{F}_{213} \otimes 1+\mathbf{F}_{21} \otimes \mathbf{F}_{1}+q \mathbf{F}_{1} \otimes \mathbf{F}_{12}+1 \otimes \mathbf{F}_{213} . \\
\Delta_{q} \mathbf{F}_{231}=\mathbf{F}_{231} \otimes 1+q^{2} \mathbf{F}_{12} \otimes \mathbf{F}_{1}+q \mathbf{F}_{1} \otimes \mathbf{F}_{21}+1 \otimes \mathbf{F}_{231} . \\
\Delta_{q} \mathbf{F}_{321}= \\
\mathbf{F}_{321} \otimes 1+q^{2} \mathbf{F}_{21} \otimes \mathbf{F}_{1}+q^{2} \mathbf{F}_{1} \otimes \mathbf{F}_{21}+1 \otimes \mathbf{F}_{321} .
\end{gathered}
$$

Finally, one can also define a one-parameter family of ordinary Hopf algebra structures on FQSym, by restricting formula (162) for $\Delta_{q}$ to connected permutations $\sigma$, and requiring that $\Delta_{q}$ be an algebra homomorphism. Then, for $q=0, \Delta_{q}$ becomes cocommutative, and it is easily shown that the resulting Hopf algebra is isomorphic to SSym.

However, it follows from [8 that for generic $q$, the Hopf algebras defined in this way are all isomorphic to FQSym. This suggests to interpret FQSym as a kind of quantum group: it would be the generic element of a quantum deformation of the enveloping algebra $\mathfrak{S S y m}=U(L)$. Similar considerations apply to various examples, in particular to the Loday-Ronco algebra PBT, whose commutative version 
obtained in 22 can be quantized in the same way as QSym, by means of $q$-commuting variables [23].

There is another way to obtain QSym from FQSym: it is known [8] that QSym is isomorphic to the image of $\operatorname{FQSym}(A)$ in the hypoplactic algebra $\mathbb{K}\left[A^{*} / \equiv_{H}\right]$. One may then ask whether there exists a $q$-analogue of the hypoplactic congruence leading directly to $Q S y m_{q}$.

Recall that the hypoplactic congruence can be presented as the bi-sylvester congruence:

$$
\begin{aligned}
& b v c a \equiv b v a c, \text { with } a<b \leq c, \\
& c a v b \equiv a c v b, \text { with } a \leq b<c,
\end{aligned}
$$

and $v \in A^{*}$.

A natural $q$-analogue, compatible with the above $q$-commutation is

$$
\begin{aligned}
& b v c a \equiv_{q H} \quad q \text { bvac, with } a<b \leq c, \\
& c a v b \equiv_{q H} \quad q a c v b, \text { with } a \leq b<c,
\end{aligned}
$$

and $v \in A^{*}$. Then, we have

Theorem 6.3. The image of $\mathbf{F Q S y m}(A)$ under the natural projection $\mathbb{K}\langle A\rangle \rightarrow$ $\mathbb{K}\langle A\rangle / \equiv_{q H}$ is isomorphic to QSym ${ }_{q}$ as an algebra, and also as a twisted Hopf algebra for the coproduct $A \rightarrow A^{\prime} \dot{+} A^{\prime \prime}, A^{\prime}$ and $A^{\prime \prime}$ being q-commuting alphabets.

Moreover, it is known that if one only considers the sylvester congruence

$$
c a v b \equiv_{S} a c v b
$$

the quotient $\mathbf{F Q S y m}(A)$ under the natural projection $\mathbb{K}\langle A\rangle \rightarrow \mathbb{K}\langle A\rangle / \equiv_{S}$ is isomorphic to the Hopf algebra of planar binary trees of Loday and Ronco [19, 13. The previous construction provides natural twisted $q$-analogs of this Hopf algebra. Indeed, let the $q$-sylvester congruence $\equiv_{q S}$ be

$$
c a v b \equiv_{q S} \quad q a c v b, \text { with } a \leq b<c .
$$

Then, since this congruence is compatible with the $q$-commutation, we have

Theorem 6.4. The image of $\mathbf{F Q S y m}(A)$ under the natural projection $\mathbb{K}\langle A\rangle \rightarrow$ $\mathbb{K}\langle A\rangle / \equiv_{q S}$ is a twisted Hopf algebra, with basis indexed by planar binary trees.

\section{ACKNOWLEDGEMENTS}

This project has been partially supported by EC's IHRP Programme, grant HPRNCT-2001-00272, "Algebraic Combinatorics in Europe". The authors would also like to thank the contributors of the MuPAD project, and especially of the combinat part, for providing the development environment for this research. 


\section{REFERENCES}

[1] M. Aguiar, N. Bergeron, and F. Sottile, Combinatorial Hopf Algebra and generalized Dehn-Sommerville relations, preprint ArXiv:math.CO/0310016.

[2] M. Aguiar and F. Sottile, Cocommutative Hopf algebras of permutations and trees, J. Alg. Comb. 22 (2005), no. 4, 451-470.

[3] F. Bergeron, G. Labelle, and P. Leroux, Combinatorial Species and Tree-Like Structures, Encyclopedia of Mathematics and its Applications, Cambridge 67, 1998.

[4] N. Bergeron, C. Reutenauer, M. Rosas, and M. Zabrocki, Invariants and coinvariants of the symmetric group in noncommuting variables, preprint ArXiv:math.CO/0502082

[5] C. Brouder and A. Frabetti, Renormalization of QED with planar binary trees, Eur. Phys. J. C, 19 (2001), 715-741.

[6] C. Brouder and R. OeckL, Quantum groups and quantum field theory: I. The free scalar field, preprint ArXiv:hep-th/0208118

[7] A. Connes and D. KREIMER, Hopf algebras, renormalization and noncommutative geometry, Comm. Math. Phys., 199 (1998), 203-242.

[8] G. Duchamp, F. Hivert, and J.-Y. Thibon, Noncommutative symmetric functions VI: free quasi-symmetric functions and related algebras, Internat. J. Alg. Comput. 12 (2002), 671-717.

[9] I.M. Gelfand, D. Krob, A. Lascoux, B. Leclerc, V. S. Retakh, and J.-Y. Thibon, Noncommutative symmetric functions, Adv. in Math. 112 (1995), 218-348.

[10] I. Gessel, Noncommutative generalization and q-analog of the Lagrange inversion formula, Trans. Amer. Math. Soc. 257 (1980), no. 2, 455-482.

[11] R. Grossman and R. G. Larson, Hopf-algebraic structure of families of trees, J. Algebra 126 (1989), n. 1, 184-210.

[12] F. Hivert, Combinatoire des fonctions quasi-symétriques, Thèse de Doctorat, Marne-LaVallée, 1999.

[13] F. Hivert, J.-C. Novelli and J.-Y. Thibon. The algebra of binary search trees, Theoretical Computer Science, to appear.

[14] F. Hivert, J.-C. Novelli and J.-Y. Thibon. Commutative Hopf algebras of permutations and trees, preprint ArXiv:math.CO/0502456

[15] A. Joyal, Une théorie combinatoire des séries formelles, Adv. Math. 42, (1981), 1-82.

[16] D. Krob and J.-Y. ThiBon, Noncommutative symmetric functions IV: Quantum linear groups and Hecke algebras at $q=0$, J. Alg. Comb. 6 (1997), 339-376.

[17] B. Leclerc, T. Scharf and J.-Y. Thibon, Noncommutative cyclic characters of symmetric groups, Jour. Comb. Theory Ser. A 75 (1996), 55-69.

[18] L. Li and P. Zhang, Twisted Hopf algebras, Ringel-Hall algebras, and Green's categories, J. of Algebra 231 (2000), 713-743.

[19] J.-L. Loday And M.O. Ronco, Hopf algebra of the planar binary trees, Adv. Math. 139 (1998) n. 2, 293-309.

[20] I.G. Macdonald, Symmetric functions and Hall polynomials, 2nd ed., Oxford University Press, 1995.

[21] C. Malvenuto and C. Reutenauer, Duality between quasi-symmetric functions and the Solomon descent algebra, J. Algebra 177 (1995), 967-982.

[22] J.-C. Novelli and J.-Y. ThiBon, An algebra of parking functions, FPSAC'04, Vancouver, 2004.

[23] J.-C. Novelli and J.-Y. ThiBon, Parking functions and descent algebras, preprint ArXiv:math.CO/0411387

[24] J.-C. Novelli and J.-Y. Thibon, Construction de trigèbres dendriformes, C. R. Acad. Sci, Paris, Sér. I, 342, (2006), 365-369.

[25] J.-C. Novelli, J.-Y. Thibon, and N.M. ThiÉRy, Algèbres de Hopf de graphes, C. R. Acad. Sci., Paris, Sér. A, 339, vol. 9, (2004), 607-610. 
[26] J.-C. Novelli and J.-Y. ThiBon, Noncommutative Symmetric Functions and Lagrange Inversion, preprint ArXiv:math.CO/0512570

[27] S. Poirier and C. Reutenauer, Algèbre de Hopf des tableaux, Ann. Sci. Math. Qébec 19 (1995), 79-90.

[28] C. Reutenauer, Free Lie algebras, Oxford, 1993.

[29] B. Sagan and M. Rosas, Symmetric functions in noncommuting variables, Trans. Amer. Math. Soc., to be published.

[30] T. Scharf and J.-Y. Thibon, A Hopf-algebra approach to inner plethysm, Adv. in Math. 104 (1994), 30-58.

[31] N. J. A. SloAne, The On-Line Encyclopedia of Integer Sequences, http://www.research.att.com/ njas/sequences/

[32] R. P. Stanley, Enumerative combinatorics, vol. 2, Cambridge University Press, 1999.

[33] J.-Y. Thibon and B.C.V. Ung, Quantum quasi-symmetric functions and Hecke algebras, Journal of Physics A 29 (1996), 7337-7348.

Institut Gaspard Monge, Université de Marne-la-Vallée, 5 Boulevard Descartes, Champs-Sur-Marne, 77454 Marne-la-Vallée Cedex 2, FRANCE

E-mail address, Florent Hivert: hivert@univ-mlv.fr

E-mail address, Jean-Christophe Novelli: novelli@univ-mlv.fr

E-mail address, Jean-Yves Thibon: jyt@univ-mlv.fr 